\title{
Estrategias dirigidas a la promoción de manualidades generadas en el programa creación, recreación y producción
}

\author{
Strategies aimed at promoting crafts generated in the creation, recreation and \\ production program
}

Joselin Beatriz Díaz Ortiz

joselindiaz25.07@gmail.com

Código ORCID: 0000-0001-8196-9814

Ministerio del Poder Popular para la Educación, Venezuela
Yarelis del Carmen Escorcia Arrieta

yescorcia54@gmail.com

Código ORCID: 0000-0002-7016-4970

Ministerio del Poder Popular para la Educación, Venezuela

La presente investigación tuvo como objetivo diseñar estrategias dirigidas a la promoción de manualidades generadas en el programa de creación, recreación y producción de la Escuela Técnica San José de Calasanz. Se fundamentó en los principios de mercadeo de Dvoskin y la teoría del desarrollo cognitivo de Piaget. Metodológicamente se desarrolló mediante el enfoque cuantitativo, obedeciendo a una investigación de tipo descriptiva con un diseño de campo. La población estuvo conformada por veinte docentes y la muestra la representaron seis (6) profesores. La técnica fue la encuesta y el instrumento un cuestionario dicotómico constituido por doce ítems. Una vez obtenida y procesada la información, se concluyó que se deben implementar las estrategias de marketing que fueron desarrolladas y propuestas en esta investigación, dentro de las cuales destacan las de publicidad y las de promoción de ventas..

Palabras clave:

Estrategias; promoción; manualidades

ABSTRACT

This research aimed to design strategies aimed at promoting crafts generated in the creation, recreation and production program of the San José de Calasanz Technical School. It was based on Dvoskin's marketing principles and Piaget's theory of cognitive development. Methodologically it was developed using the quantitative approach, obeying descriptive research with a field design. The population was made up of twenty teachers and the sample was represented by six (6) teachers. The technique was the survey and the instrument was a dichotomous questionnaire consisting of twelve items. Once the information was obtained and processed, it was concluded that the marketing strategies proposed in this research should be implemented, among which those of advertising and sales promotion stand out. 


\section{INTRODUCCIÓN}

La educación venezolana actualmente está generando una serie de transformaciones, tanto en la estructura curricular como en las estrategias que deben aplicar los docentes, las cuales tienen como finalidad promover el desarrollo de las diferentes competencias y habilidades en los estudiantes, potenciando así todas las capacidades humanas como ser antropológico con carácter reflexivo analítico dentro de su propia concepción.

El desarrollo de dichas competencias y habilidades permiten al estudiante identificar y argumentar los conocimientos, planificar y consolidar el proceso creativo, reconocer las habilidades perceptivas-analíticas y evaluar con operatividad la integración de un determinado trabajo que consigue perfeccionar las habilidades manuales mediante la praxis con el fin de fabricar productos e incorporarse dentro del mercado productivo y laboral que demanda la economía global. Apuntando en esta dirección se puede decir que el desarrollo de dichas actividades manuales, se traducen en un oficio que les permita a los estudiantes incorporarse fácilmente dentro de un mercado laboral y obtener beneficios.

El problema radica en que el sistema educativo está enseñando todas estas actividades por medio de la imitación, lo que ha afectado negativamente la originalidad y la creatividad de los que participan en el hecho. El espacio para la improvisación y la búsqueda de otras alternativas y propuestas a través de la libertad para crear es limitado. La imitación va acostumbrando al estudiante a aplicar fórmulas y a memorizar conceptos que olvidará una vez culminado el examen.

Resulta oportuno afirmar que el ser humano tiene la capacidad de aprender de forma continua, y esta capacidad se desaprovecha cuando, en los procesos pedagógicos, no se implementan las estrategias adecuadas para propiciar el aprendizaje significativo que el educando pueda utilizar en su cotidianidad. Tanto ha sido el impacto de esta problemática, que los propios alumnos se niegan a aprender de una forma distinta y a poner en práctica lo aprendido.

En el caso de las manualidades, es relevante decir que representan una estrategia de enseñanza que permite promover la creatividad, el aprendizaje significativo y útil a partir del desarrollo de destrezas manuales y artísticas para la elaboración de un producto y el perfeccionamiento de las habilidades tanto motoras como cognitivas.

Es por ello que el Ministerio del Poder Popular para La Educación ofrece la participación a través de grupos de Creación, Recreación y Producción (CRP) que están paralelamente relacionados con la práctica y las vivencias en las que los estudiantes amplían todas sus capacidades, inclinaciones o vocaciones. Para ampliar este tema, es importante tomar en cuenta las diferentes leyes o decretos existentes en Venezuela en torno a la producción, educación, manualidades, entre otros.

En primer lugar, se tiene la Constitución de la República Bolivariana de Venezuela (1999) en su artículo 112 donde establece: "toda persona puede dedicarse libremente a la actividad económica de su preferencia, sin más limitaciones que las previstas en esa constitución". Mientras en el artículo 308, "el estado protegerá y promoverá la pequeña y mediana industria, las cooperativas, cajas de 
ahorro, así como también la empresa familiar, la microempresa y también otra forma de asociación comunitaria para el trabajo, el ahorro y el consumo" Lo que significa que el Estado reconoce y apoya a las pequeñas y medianas industrias; por lo cual se puede argumentar que los pequeños productores o artesanos son tomados en cuenta en la Carta Magna.

Asimismo, se tiene que la Ley Orgánica de Educación (2009) en su artículo 4 establece: "La educación como derecho humano y deber social fundamental orientada al desarrollo del potencial creativo de cada ser humano en condiciones históricamente determinadas (...)". Y la Ley para la Activación y el Fortalecimiento de la Producción Nacional (2016) cuyo objeto es "promover la producción nacional de bienes y servicios, la seguridad alimentaria y el crecimiento económico, estimular las inversiones en la actividad productiva...". Esto significa, la educación no está alejada de esta realidad donde ella forma parte del aparato productivo y económico del País, pues desde las instituciones educativas también se pueden generar recursos para su auto sustento y así satisfacer algunas necesidades.

Por lo tanto, en Venezuela se establecen, a través de la transformación curricular para la Educación Media General, estrategias que impulsan la creación de estos Grupos Estables dentro de las instituciones educativas con el fin de que los jóvenes aprendan un oficio y puedan desarrollar sus habilidades para luego ponerlas en práctica. $A$ través del ente rector de la educación, el Ministerio del Poder Popular para la Educación (2017) se estableció que:
Los Grupos de Creación, Recreación

y Producción, como política educativa en el marco de la transformación pedagógica, forma parte de un nuevo Plan de Estudios a nivel de Educación Media General su propósito es generar espacios solidarios, de construcción colectiva para una cultura de paz, permitiendo la participación protagónica de los y las estudiantes, desde lo académico, cultural, deportivo y productivo en el área creadora de su interés. (p.2)

Sin embargo, en medio de este plan de grupos de creación, recreación y producción se puede observar que los profesores encargados de llevar a cabo estas actividades no están desarrollando las estrategias adecuadas para la promoción y comercialización de los objetos que se producen en los grupos ni tampoco están aportando algo al desarrollo económico.

Por el contrario, existen numerosos grupos y proyectos de esta misma índole que si están produciendo y dando excelentes resultados en su gestión y que se pueden mencionar como antecedentes de esta investigación. Tal es el caso de Gutiérrez (2018) en su investigación "Escuela Cruz Felipe Iriarte desarrolla grupos de creación, recreación y producción" señala que la escuela "...se encuentra desde hace varios meses trabajando en la ejecución de diferentes grupos de creación, recreación y producción, para todo el alumnado de educación media (...) los grupos de interés han estado desarrollándose con entusiasmo por parte de los estudiantes" (p.27). Allí se 
cuenta con grupos de fútbol de playa, voleibol de playa, repostería, peluquería, manos a la siembra, dibujo, César Rengifo y música" y tales grupos tienen una conformación aproximada entre 20 y 30 estudiantes.

De igual forma, el autor expresó que esperan desarrollar el reto estudiantil de matemáticas donde se incorporará la parte numérica a cada uno de los grupos. Por ejemplo, explicarán la matemática en las recetas, elaboración de desinfectantes, desodorantes y proyectos de energía eólica. "La elaboración de estos productos y preparaciones una vez realizadas, los alumnos las venden y con el dinero recaudado pueden comprar nuevamente los materiales e ingredientes para la creación de nuevos productos (p. 28).

Otra investigación relevante fue la realizada por Otero (2017) titulada "Material de provecho como herramienta pedagógica en la educación básica como guía práctica en la realización de manualidades" donde se expresa lo siguiente: "La Tierra cada día se está deteriorando (...) El desarrollo de una conciencia ecológica comienza en la familia y luego en la escuela" (p.18). Es por ello que este estudio tuvo como objetivo proponer una guía práctica para la aplicación del material de provecho como herramienta pedagógica en la realización de manualidades. Los resultados arrojados apuntaron a la necesidad de diseñar una guía práctica para la aplicación del material de provecho en la producción de manualidades en la institución.

Considerando lo descrito anteriormente y mediante la observación directa se puede evidenciar que en la Escuela Técnica "San
José de Calasanz", ubicada en la ciudad de Valencia del estado Carabobo, Venezuela, hay una deficiencia en la implementación de estrategias promocionales que impulsen la comercialización de las manualidades creadas por los estudiantes. Entre estas estrategias promocionales se pueden mencionar las redes sociales, marketing digital, publicidad, ventas personales y otras herramientas de publicidad y mercadeo que propicien la difusión de estas actividades.

Esta difusión, además de proporcionar un beneficio económico en el caso de que las herramientas mencionadas se implementen de manera eficiente, le permite al estudiante dar a conocer su talento, obteniendo así el reconocimiento y la motivación que le permita impulsar y seguir perfeccionando sus talentos $y$ habilidades a través de la aplicación de los conocimientos adquiridos y las destrezas manuales sobre el marco del hacer.

Cabe destacar que esta manera de aprendizaje significativo está vinculada con lo que indica Piaget (1964), en torno al desarrollo mental en el individuo: "la profunda unidad de los procesos que, partiendo de la construcción del universo práctico, debido a la inteligencia sensoriomotriz del lactante, desemboca en la reconstrucción del mundo mediante el pensamiento hipotético-deductivo" (p.93). De manera tal, que el protagonista del aprendizaje es el propio aprendiz, y no sus tutores ni sus maestros. Este planteamiento es llamado enfoque constructivista, y enfatiza la autonomía de la que disponen los individuos a la hora de interiorizar todo tipo de conocimientos; según este enfoque, es la persona quien sienta las bases de su 
propio conocimiento, dependiendo de cómo organiza e interpreta la información que capta del entorno.

De igual forma, durante el estudio se observó que la institución está regida por una organización tradicional, de estructura jerárquica rígida, procesos gerenciales rigurosos con poca importancia hacia el trabajo en equipo, motivo por el cual se demuestra que no han sido suficientes los esfuerzos realizados por el gerente y los docentes, para establecer de forma estable y permanente ese vínculo interpersonal entre los actores involucrados para establecer la convivencia.

Asimismo, la falta de integración de la comunidad con la institución se notó aislada de los proyectos que se están implementando actualmente en el plantel, lo cual exige la participación de todos con el propósito de que los estudiantes, docentes y miembros de dicha comunidad conozcan la realidad y se vean en la obligación de ser corresponsables en la obtención del beneficio común.

Es importante tener presente que "el sujeto almacena su conocimiento de la realidad mediante distintos tipos de entidades de diferente naturaleza. En ellas se pueden distinguir los esquemas, los conceptos y las representaciones" (Deval, 1997, p.24). Posiblemente estos tres últimos elementos constituyan instrumentos claves para la construcción de su conocimiento y consolidación de competencias las cuales le ayuden a salir adelante en las actividades inherentes en su vida diaria. Además, hoy en día, la forma de construir el conocimiento es otra, se vive una realidad donde la información tiene una gran velocidad y puede llegar casi instantáneamente a lugares recónditos del planeta; pues desde la perspectiva constructivista, "el desarrollo del individuo es un proceso de construcción y organización de los conocimientos" (Raybal y Rieunier, 2010, p.122).

Es por ello, que el factor importante para el avance de la institución son las relaciones interpersonales, las cuales juegan un papel fundamental en el desarrollo integral de la persona. A través de ellas, el individuo obtiene importantes refuerzos sociales del entorno más inmediato que favorecen su adaptación al mismo; así como también, a través de experiencias enriquecedoras el estudiante podrá adquirir y desarrollar más habilidades. En tal sentido, se presenta la siguiente interrogante que guió el estudio: ¿Cuáles son las estrategias de promoción que logren facilitar las ventas de las manualidades que producen los estudiantes adscritos al programa de Grupos Estables?

Para ellos se estableció como objetivo general diseñar estrategias dirigidas a la promoción de manualidades generadas en el Programa Creación, Recreación y Producción de la Escuela Técnica "San José de Calasanz". $Y$ para alcanzar el mismo, se fijaron como objetivos específicos, en primer lugar diagnosticar los métodos de difusión que emplean los estudiantes en la promoción de manualidades generadas por el Programa Creación, recreación y producción; seguidamente, determinar las estrategias aplicadas en la promoción de manualidades creadas por los estudiantes de educación media general y así elaborar estrategias para 
la promoción de manualidades generadas en el Programa Creación, recreación y producción en dicha Escuela Técnica.

Es importante señalar, la presente investigación permitió demostrar una gama de beneficios tanto a los estudiantes como a la institución, a través de la obtención de conocimientos para ejecutar diversas formas de promoción y finalmente crear productos y servicios que puedan organizarse por medio de distintas estrategias la cual, consiste en agrupar equipos conformados por personas de un mismo oficio, que hacen el ejercicio de producir ciertos artículos.

Siguiendo este mismo orden de ideas, el estudio se justificó porque presentó estrategias que ofrecieron a los estudiantes la oportunidad de obtener un apoyo socioeconómico y de sustentabilidad enmarcado dentro de sus necesidades como individuo, favoreciendo a la institución y su ámbito socioeducativo. Manteniendo presente, que una estrategia es un "procedimiento organizado, formalizado y orientado a la obtención de una meta claramente establecida. Su aplicación en la práctica diaria requiere del perfeccionamiento de procedimientos y de técnicas cuya elección detallada y diseño son responsabilidad del docente" (Montealegre, 2016, p. 20).

En cuanto a la inclusión de la comunidad, permitió promover lazos de afiliación entre los partícipes, incidir en nuevos proyectos para tratar de minimizar las carencias que se encuentran visibles dentro de un espacio determinado, adoptando una postura de organización y planificación regida bajo un sistema cooperativo.

Asimismo, a través de este diseño se buscó impulsar, dentro de los órganos garantes de la Educación Universitaria, nuevos estudios vinculados a la investigación de estrategias relacionados a la promoción, que de igual forma represente un aporte novedoso ante la línea de investigación Turismo, Cultura y Mercadeo, siendo la misma una manifestación para la formación integral del hombre y mujer perteneciente a la sociedad venezolana.

Del mismo modo, tomando en consideración el Programa de Creación, Recreación y Producción (CRP) se pueda crear una nueva cultura escolar con relación al carácter humano entrelazado al ejercicio pleno del trabajo. Siendo éste un área de formación establecido dentro del Currículo Bolivariano, que tiene como base un enfoque teórico-práctico vinculado al desarrollo de habilidades psicomotoras las cuales se relacionan través de la praxis.

Para finalizar este estudio está justificado porque buscó promover el proceso social del trabajo, superando la relación mercantilista bajo condiciones que permitan potenciar participaciones en producciones de bienes y servicios en relación a la comunidad y sociedad en general. Según Méndez (2016) el Programa de Creación, Recreación y Producción "tiene una fundamentación para el Trabajo Colectivo y Cooperativo en el desarrollo de experiencias sobre el marco de hacer, el ser, el sentir y el convivir". (p. 4) De esta manera los grupos estables implican una nueva responsabilidad para quienes se encarguen de coordinarlos, también significan entrar en una dinámica de horarios que es compleja por la naturaleza en la que se viene desarrollando el bachillerato en Venezuela; donde los profesores trabajan en dos y hasta en tres 
instituciones para llevar una mejor calidad de vida.

Aunque el mayor temor, es que los grupos estables se conviertan en una imposición o en una decisión inconsulta, como de seguro está pasando en muchas instituciones, dada la aceleración temporal con la que ha venido acompañada la transformación curricular. Algunos piensan que vienen a ser el equivalente a la Educación para el Trabajo, si bien la producción de bienes y servicios es un área, no es la única. Los grupos estables son una opción educativa alternativa, una oportunidad para sincerar el currículo a la luz de las necesidades y talentos que tienen los estudiantes; son, en cierta medida, una oportunidad democrática para que cada joven descubra y comparta sus talentos. Por ello, es un gran impulso para muchos jóvenes que se encuentran en la etapa de descubrirse, de indagar y aprender sobre lo que pueden dar de sí.

\section{MÉTODO}

Una vez descrito el problema de investigación y planteados los objetivos que se persiguen, resulta relevante exponer la metodología implementada. En este punto resulta pertinente citar a Balestrini (2006) quien afirma que la metodología corresponde al "conjunto de procedimientos lógicos, tecno-operacionales implícitos en todo proceso de investigación, con el objeto de ponerlos de manifiesto y sistematizarlos; a propósito de permitir descubrir y analizar los supuestos del estudio y de reconstruir los datos" (p.44). Es decir, la metodología responde al conjunto de acciones destinadas a describir y analizar a fondo el problema planteado, a través de procedimientos específicos que incluye las técnicas de observación y recolección de datos, y determina el cómo se realizará el estudio.

De este modo, en cuanto a la ruta metodológica que se asumió en la investigación se puede decir que estuvo enmarcada en el paradigma positivista de carácter cuantitativo que Hernández, Fernández y Baptista (2010), definen como "aquella que usa la recolección de datos para probar hipótesis, con base en la medición numérica y el análisis estadístico, para establecer patrones de comportamiento y probar teorías" (p.4). Es por ello, que el enfoque de la investigación fue cuantitativo, ya que sus resultados se analizaron a partir de frecuencias y porcentajes apoyados en la estadística descriptiva.

Ahora bien, respecto al tipo de investigación, se refiere a la clase de estudio que se realiza y además orienta la finalidad general del estudio y la manera de recoger la información o datos necesarios. En las investigaciones de tipo descriptiva, como es el caso, llamadas también investigaciones diagnósticas, consiste fundamentalmente en caracterizar un fenómeno o situación concreta indicando sus rasgos más peculiares 0 diferenciadores. Según Rísquez, Fuenmayor y Pereira, (1999) exponen en cuanto a este tipo de investigación:

(...) tiene una mayor profundidad, dado que va más allá de la exploración porque con esta se busca medir las variables que intervienen en el estudio, de acuerdo con sus características, 
actitudes y del comportamiento de las unidades investigadas. Respondiendo a cuenta, a qué medida o como se produce el problema de estudio. (p. 39)

Tomando en cuenta todo esto, se puede decir que esta investigación fue de tipo descriptiva, ya que para recopilar los datos necesarios se identificaron las distintas situaciones, hechos y características del problema mediante una serie de preguntas estructuradas, realizadas a las cinco personas pertenecientes a la muestra extraída. Es por ello que, esta investigación se clasificó como una investigación con un diseño de campo o no experimental, ya que se utilizaron datos primarios recopilados directamente desde el lugar donde se encuentra el problema en este caso en la Escuela Técnica "San José de Calasanz".

Del mismo modo, la población de estudio se conformó por una selección de individuos que son el foco principal de una investigación, esto quiere decir que se realizan con el fin de solucionar un problema que afecta a la población. Arias (2001) la define como: un conjunto finito o infinito de elementos con características comunes para los cuales serán extensivas las conclusiones de la investigación. Ésta queda delimitada por el problema y por los objetivos del estudio (p.81).

En efecto, para la presente investigación se tomó como población una cantidad de veinte (20) docentes pertenecientes a la Escuela Técnica San José de Calasanz de los cuales se extrajo una muestra para poder trabajar y recolectar información más precisa acerca del objeto estudiado. La selección de la muestra es un proceso en el que el investigador selecciona directa e intencionadamente a los individuos de la población. Es decir, la muestra es una porción de la población y debe poseer las mismas características para poder generalizar los resultados obtenidos de la muestra hacia la población con mínimo margen de error. Para efectos de la presente investigación, se extrajo una muestra de seis (6) docentes que representaron el 30\% de la población la cual es de tipo intencional. En lo que se refiere al tamaño de la muestra, cabe considerar algunas opiniones de expertos en este tópico como Palella y Martins (2011), quienes expresan que

(...) diversos autores coinciden
en señalar que una muestra de
$10 \%, 20 \%, 30 \%$ o $40 \%$ puede ser
representativa de la población,
acorde con su tamaño; con la
acotación de que si dentro de la
población coexisten sujetos que
posean distintas características
deben ser representados en
idénticas proporciones que
poseen en ésta. (p. 54)

Así pues, en lo que se refiere a las técnicas, se aplicó una encuesta que permitió obtener información directa de los individuos que componen la muestra. A través de ella se obtuvo un contacto cara a cara y personal con la población de estudio. Para realizarles la encuesta fue necesario implementar un instrumento de recolección de datos que en este caso fue un cuestionario dicotómico, constituido por doce (12) ítems de preguntas cerradas y 
respuestas múltiples que permitió obtener datos específicos de interés y gran aporte a la investigación.

Cabe acotar que antes de la aplicación del instrumento fue necesario evaluar su validez, la cual se refiere al grado de eficiencia en que el instrumento mide las variables que el investigador desea analizar. Considerando lo indicado por Sabino (2000) "es la eficacia con que un instrumento mide lo que se pretende. Lo cual permite concluir que la validez de un instrumento se encuentra relacionada directamente con el objetivo del instrumento" (p.30).

Por esta razón, dicha validez fue revisada mediante el juicio de expertos; se le presentó el cuestionario a dos especialistas en el área y profesores de la mención quienes lo revisaron, realizaron sus observaciones y concluyeron que el instrumento podía ser aplicado. En el caso de la confiabilidad del mismo, se aplicó el método Kuder Richarson (formula KR-20), por cada uno de los ítems con respuestas de tipo dicotómica. El resultado fue 0,61 , lo cual indica que es un coeficiente alto y confiable. Al respecto, Hernández et al. (2010), alegan que la confiabilidad de un instrumento:

(...) se refiere al grado en la cual su aplicación repetida al mismo sujeto produce iguales resultados. Adicionalmente exponen que existen diversos procedimientos para calcular la confiabilidad de un instrumento de medición. Todos utilizan fórmulas que producen coeficientes de confiabilidad y que pueden oscilar entre 0 y 1 , es decir, cuanto más se acerque a cero mayores errores

habrá en la medición. (p. 215)

Una vez confirmada la confiabilidad del instrumento, se procedió a realizar la recolección de datos con el fin de diagnosticar los métodos de difusión que emplean los estudiantes en la promoción de sus manualidades y así determinar las estrategias aplicadas para darlas a conocer, y finalmente promocionarlas como producto del Programa Creación, Recreación y Producción de la Escuela Técnica "San José de Calasanz".

\section{RESULTADOS Y DISCUSIÓN}

Una vez realizado el diagnóstico se procedió con la interpretación de los resultados que se alcanzaron mediante la aplicación de los instrumentos a seis docentes guías de la Escuela Técnica "San José de Calasanz", ubicada en Valencia, Estado Carabobo. Los mismos fueron organizados para efectos de esta producción, en dos dimensiones descritas a continuación.

En primer lugar, se tiene la Dimensión Estrategia conformada por cuatro ítems: En el Ítem 1 se pregunta al participante si ¿Sus estudiantes emplean algunas de las redes sociales para promover un producto? El 66,7 por ciento de las personas respondió de forma afirmativa mientras el 33,3 por ciento respondió de manera negativa. Seguidamente en el ítem 2 ¿Los participantes del programa de manualidades conocen estrategias publicitarias? Los informantes señalaron que el 66,7 por ciento no tienen conocimiento alguno; mientras el 33,3 por ciento si conocen. En el ítem 3 se 
preguntó si ¿Promocionan de manera voluntaria las manualidades? Manifestando los encuestados que el 50 por ciento de sus estudiantes lo hacen y el otro porcentaje no. Finalmente, para esta dimensión estrategias de aprendizaje, el ítem 4 consultó: ¿Los estudiantes personalmente venden sus manualidades? Confirmando que el 83,3 por ciento de ellos no lo hacen. Todo esto se muestra en la siguiente figura 1.

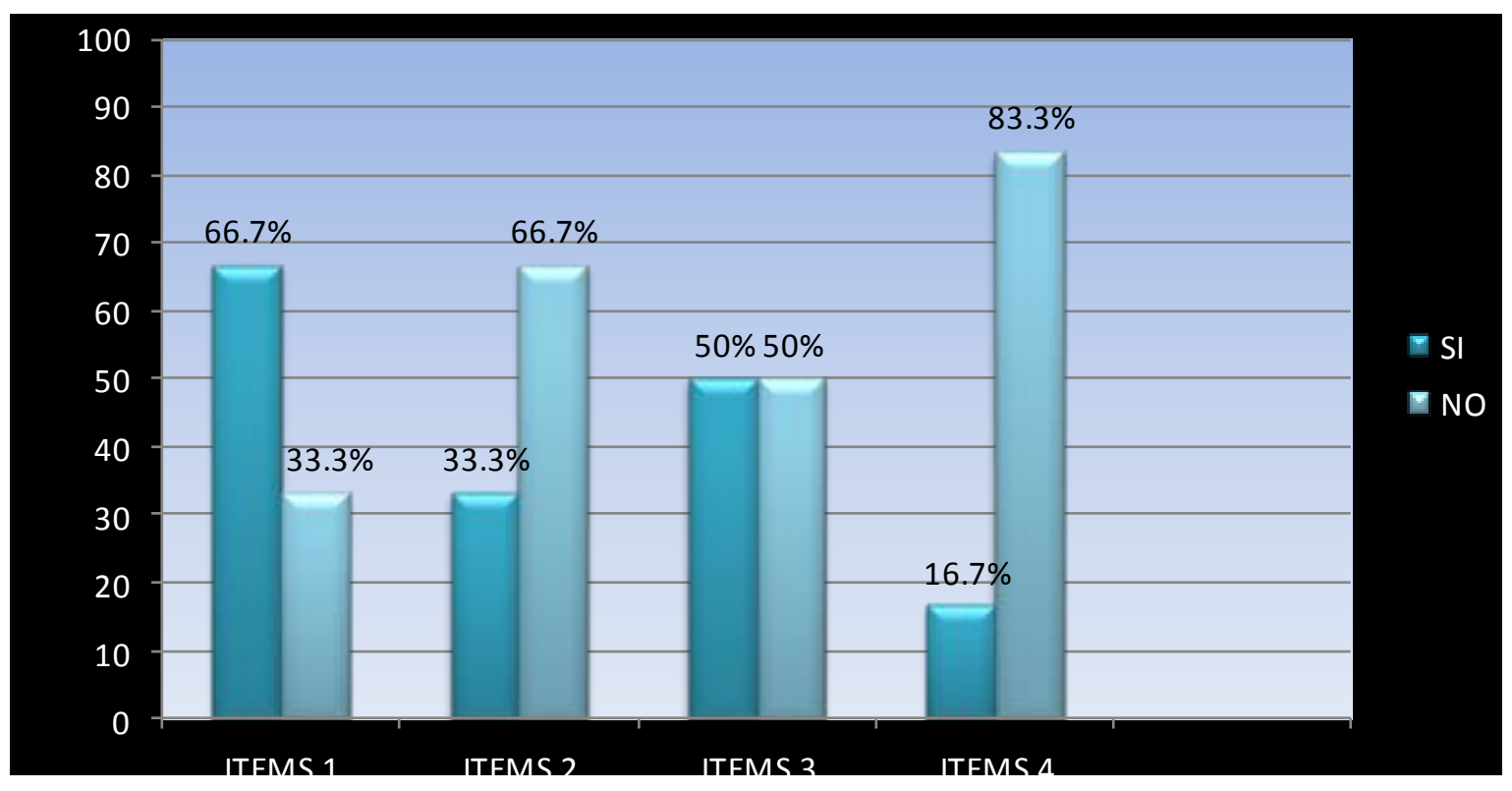

Figura 1. Dimensión estrategia. (Fuente: Díaz y Escorcia, 2020)

En síntesis, se pudo observar que, de acuerdo al instrumento aplicado en la institución, el 41,7 por ciento de los profesores manifiesta que los estudiantes conocen y emplean las diferentes estrategias que se utilizan para promocionar un producto, mientras que el 58,3 por ciento de los participantes no tienen información de dichas prácticas. Por lo cual es vital en el ámbito educativo, enseñar manejo de estrategias de publicidad y mercadeo a los estudiantes interesados en comercializar las manualidades que construyen en el grupo.
La segunda dimensión Experiencias en el Programa C.R.P., estuvo conformada por cuatro ítems que van del 5 al 8 donde se les preguntó a los docentes en primer lugar, en el ítem 5 si ¿Los participantes aplican los conocimientos aprendidos en el programa? En este caso afirmaron que el 50 por ciento de los estudiantes aplican el conocimiento aprendido. Seguidamente, en el ítem 6 ¿Los docentes facilitan los conocimientos que los participantes necesitan en su vida diaria? Destacando que el 66,7 por ciento de los docentes entrevistados consideran que si se 
están facilitando los conocimientos que el joven necesita para el desenvolvimiento en su vida; mientras un 33,3 por ciento piensa lo contrario. Por otra parte, en el ítem 7 ¿Los estudiantes desarrollan destrezas con utilidad práctica? Allí el 83,3 por ciento de los docentes informantes manifestaron que los estudiantes no desarrollas destrezas con un fin práctico para su vida cotidiana. Ya para finalizar, en el ítem 8 ¿Se direccionan los talentos que posee cada aprendiz? el 50 por ciento de los encuestados consideran que si se direccionan los talentos que posee cada estudiante y el otro 50 por ciento dice lo contrario. En la figura 2 se expresa de manera articulada cada uno de estos ítems en atención a la dimensión experiencia en el programa C.R.P.

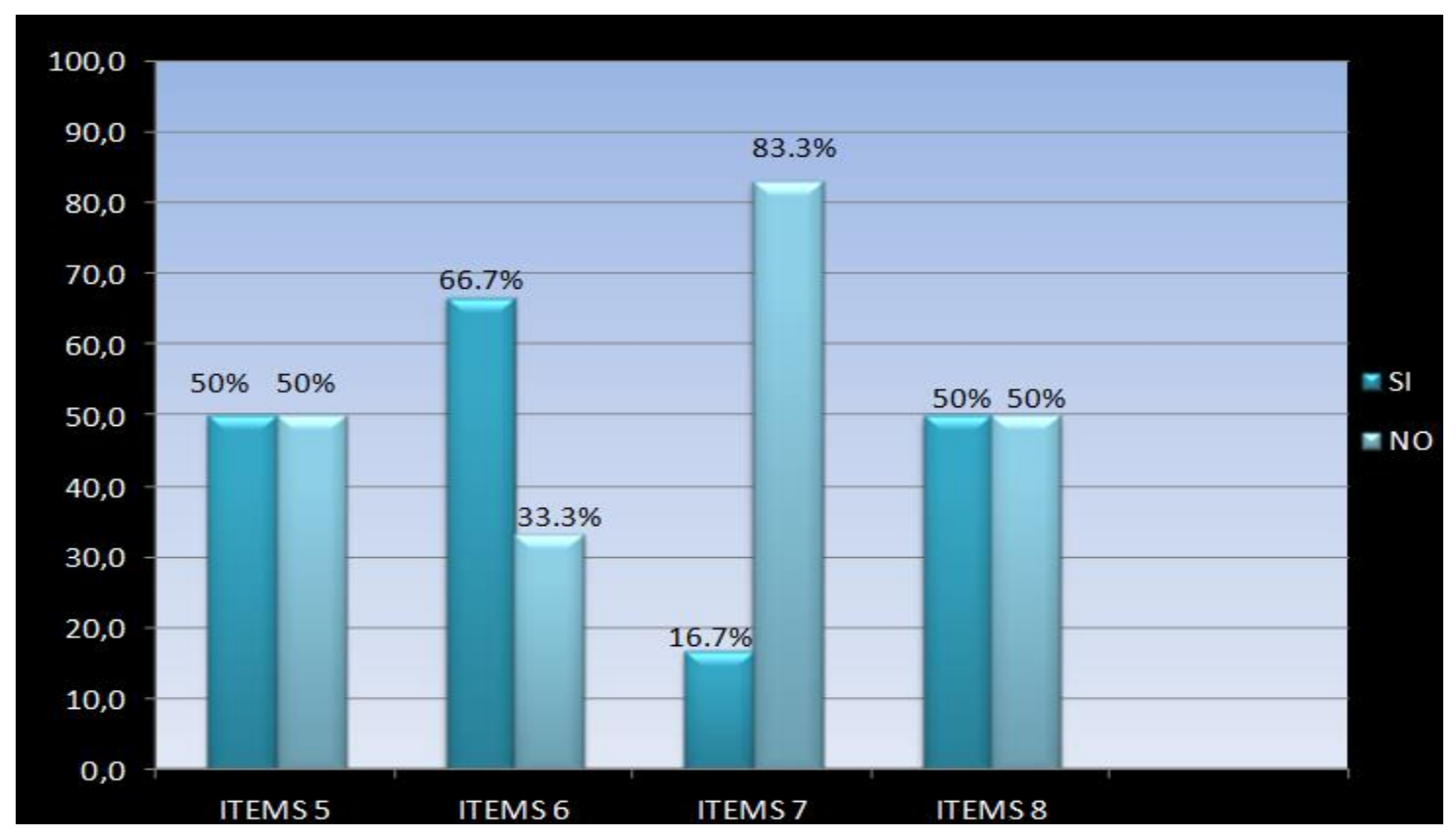

Figura 2. Dimensión experiencia en el programa C.R.P. (Fuente: Díaz y Escorcia, 2020)

En vista de los resultados obtenidos en la entrevista aplicada en la Escuela Técnica "San José de Calasanz", se pudo comprobar que no se realiza publicidad por ningún medio de comunicación e información de los productos que confeccionan los estudiantes ya que son muy pocos los que conocen cómo hacerlo. Se considera que los estudiantes deben aprender técnicas y estrategias para promocionar sus productos y aprovechar las habilidades manuales que tienen los estudiantes ya que son muy talentosos y además, eso puede proporcionar beneficios para la institución y la comunidad. En concordancia con Martínez (2004) el trabajo colectivo es considerado como:

(...) una actividad de ayuda técnica y organizada ejercida sobre las personas, grupos 0 comunidades, tratando que se ayuden a sí mismas con el fin de procurar su plena realización, su mejor funcionamiento social $y$ mayor bienestar. Esto se hará 
con la activación de los recursos internos y externos principalmente los ofrecidos por los servicios sociales y otras instituciones. Por lo tanto, se trata de una actividad técnica que se estudia, se aprende y se enseña. (p.10)

En lo que concierne a las estrategias promocionales, los profesores afirman que los participantes no tienen conocimientos al respecto, por lo cual no saben cómo mercadear sus productos. Esto es preocupante ya que el mismo es considerado por Dvoskin (2004) como "el resultado del conjunto de elecciones, acciones y decisiones de la organización respecto a cada una de las áreas... para responder a las necesidades que plantean distintos mercados, productos $\mathrm{y}$ clientes" (p.26).

Otro aspecto que se pudo detectar en este estudio es que, según los docentes, los aprendices no aprovechan ni continúan desarrollando las cosas que aprenden en la escuela en su vida cotidiana y tampoco saben cómo llevar esos conocimientos a la práctica.

Se puede decir entonces que, en vista del problema existente, es necesario la implementación estrategias que ayuden al estudiante a contextualizar los conocimientos que adquiere en la escuela y los aproveche para su desarrollo personal. En relación al trabajo en equipo, los profesores aseguran que puede inducir a una mejor relación docente-estudiantes en conjunto con la comunidad, por lo cual es un factor que puede influir positivamente en la solución del problema planteado. Esto se explica con más detalle en el siguiente punto.

\section{CONCLUSIONES}

La presente investigación logró cumplir con los objetivos planteados. Se diagnosticaron los métodos de difusión que emplean los estudiantes en la promoción de manualidades generadas por el Programa Creación, Recreación y Producción, donde se logró evidenciar que las principales fortalezas de los participantes son las habilidades y el talento que poseen para desenvolverse en el desarrollo de una amplia gama de manualidades. Por su parte, se pudo concluir la debilidad que les afecta está en la carencia de promoción y difusión de los productos para la adquisición a terceros.

Una vez realizado el diagnóstico, se pudo determinar que las estrategias aplicadas en la promoción de manualidades son una alternativa altamente viable para convertir el programa creación, recreación y producción en una actividad productiva que genere una serie de beneficios a la comunidad estudiantil. Se llegó entonces a la conclusión de que la implementación de estrategias de publicidad y mercadeo proporcionan un amplio abanico de información, persuasión y comunicación, que pueden ayudar a promocionar las manualidades en la comunidad en general.

Entre las estrategias propuestas para cumplir con el objetivo y promocionar los productos que construyen los participantes se pueden mencionar las siguientes: La creación de una cuenta en Instagram a modo de "catálogo virtual" que esta permite dar a conocer los productos a una comunidad a nivel mundial y donde los usuarios podrán 
apreciar las creaciones de los participantes a toda hora y en cualquier momento, desde cualquier parte mediante una conexión a internet. Adicionalmente se elaboraron un conjunto de folletos tipo bolsillo que los interesados podrán llevar consigo gracias a su tamaño y que resultó ser manejable y accesible por quienes no están familiarizados con los recursos digitales. Asimismo, también se realizaron carteles publicitarios.

Otra estrategia promocional sugerida fue la difusión de los productos a través del canal de radio "La Monumental 94.1", y llegar así a una amplia audiencia porque los mensajes en radio tienen la particularidad de que, al ser oídos de forma repetida, asegura al radioescucha recordar el producto que se está publicitando.

Resulta claro que gracias al apoyo de las estrategias descritas anteriormente se pudo llegar a una audiencia mucho más representativa, lo que contribuirá de manera considerable a la difusión de este proyecto, dar a conocer el trabajo que realizan los educandos que participan en este grupo y además, repercute positivamente al incremento de las ventas, generando así un beneficio económico y posición en el mercado con la intención de crear a mediano plazo nuevas alianzas con la comunidad.

Aunado a esto se buscó la formación integral de los participantes en el proceso educativo, especialmente en el programa grupos estables de manualidades, considerando las estrategias de comercialización como planes específicos donde se toma en cuenta tanto al consumidor como la promoción de ventas de los productos. Esto con el fin de incrementar las ventas y dar a conocer los productos que se realizan, así como también impulsar la credibilidad de la institución en cuanto a la educación que brindan a sus estudiantes.

Como aporte adicional, este estudio se propone una siguiente fase donde se profundice sobre estrategias integrales de producción y comercialización, así como las tácticas de cada una de ellas que le proporcionen conocimientos y capacitación a los estudiantes para desenvolverse en campo publicitario ya sea virtual o personal.

Finalmente, respecto a las estrategias mencionadas derivadas de los resultados obtenidos en esta investigación, fueron aplicadas evidenciándose una modificación favorable en la implementación del programa Grupos Estables de la institución educativa, los cuales permitieron alcanzar grandes conocimientos a los participantes al desenvolverse en campo promocional. De igual forma, generó la participación de la comunidad en las actividades realizadas en la institución. En cuanto a los estudiantes, esta investigación creó una nueva forma de integrarse en el proceso educativo, especialmente en el programa grupos estables de manualidades, así como también la promoción de los productos creados.

En este sentido, el estudio aportó una serie de estrategias de mercadeo y promoción para beneficio de los participantes interesados en la proyección de sus manualidades con las cuales pueden obtener beneficios económicos mientras terminan su capacitación para entrar al mercado laboral o incluso para realizarlas como actividad paralela a su carrera profesional. En este trabajo también se 
contribuyó en el fortalecimiento de la relación entre comunidad e institución y satisfacción de las necesidades que se exponen dentro de la pirámide social de cada uno de los estudiantes.

A modo de cierre se puede manifestar que las actividades que tienen que ver con las manualidades capacitan a los estudiantes en el aprendizaje de un oficio que les va a permitir su incorporación al mundo del emprendimiento y obtener numerosos beneficios para su desarrollo personal.

\section{REFERENCIAS}

Arias, F. (2001). El Proyecto de Investigación. Guía para su elaboración. Venezuela: Editorial Episteme

Balestrini, M. (2006). Como elaborar un proyecto de investigación. Caracas: Consultores Asociados

Constitución de la República Bolivariana de Venezuela. Gaceta Oficial de la República de Venezuela, 36.860 (Extraordinario), Diciembre 30, 1999

Deval, J. (1997). Tesis sobre el constructivismo. En Coll. C y Gabucio, F. (Dir.) (Rodrigo, M. \& Arnay, J., Comps.) La construcción del conocimiento escolar. Vol. 2. Temas de Psicología. (1 ${ }^{a}$. ed. pp.15-33) Barcelona: Paidós

Dvosking, R. (2004). Fundamentos de marketing. Buenos Aires, Argentina: Ediciones Granica S.A

Gutiérrez, L. (2018). Escuela Cruz Felipe Iriarte desarrolla grupos de creación, recreación y producción. Recuperado de https://www.vargasesnoticia.com/escuela -cruz-felipe-iriarte-desarrolla-grupos-decreacion-recreacion-y-produccion/
Hernández, R., Fernández, T. y Baptista, P. (2010). Metodología de la Investigación. México: Editorial Ultra

Ley Orgánica de Educación. Gaceta Oficial de la República de Venezuela, 5.929 (Extraordinario), Agosto 15, 2009

Ley para la Activación y el Fortalecimiento de la Producción Nacional. Gaceta Oficial de la República de Venezuela, 40.929 (Extraordinario), Noviembre 30, 2016

Martínez, M. (2004). El trabajo social y los servicios sociales. Su concepto. Recuperado de http://biblioteca.ues.edu.sv/revistas/1080 0247-8.pdf

Ministerio del Poder Popular para la Educación (2017). Área de formación: grupos de creación, recreación y producción. Caracas, Venezuela: Viceministerio de Educación Media

Méndez, E. (2016). Grupos estables una opinión de construcción y participación estudiantil. Recuperado de http://www.movimientopedagogico.com. ve/Los-grupos-estables:-una-opcion-deconstruccion-y-participacion-estudiantiln-750.html

Montealegre, A. (2016). Estrategias para la enseñanza y el aprendizaje de las ciencias. [Libro en línea] Recuperado de http://ebookcentral.proquest.com

Otero, F. (2017). Material de provecho como herramienta pedagógica en la educación básica como guía práctica en la realización de manualidades. (Tesis de Grado de Maestría). Recuperado de http://mriuc.bc.uc.edu.ve/bitstream/hand le/123456789/7021/fotero.pdf?sequence $=1$ 
Palella, S. y Martins, F. (2010). Metodología de la investigación cuantitativa. Venezuela: Editorial FEDUPEL

Piaget, J. (1964). Seis estudios de Psicología. Barcelona: Editorial Labor S.A

Rísquez, G., Fuenmayor, E. y Pereira, B. (1999). Como hacer un Proyecto de
Investigación. Caracas, Venezuela: Ediciones Panapo

Sabino, C. (2000). El proceso de investigación. Caracas, Venezuela: Ediciones Panapo 